\title{
Coincidence and common fixed point theorems for Suzuki type hybrid contractions and applications
}

\author{
Shyam Lal Singh ${ }^{1}$, Raj Kamal ${ }^{2}$ and Manuel De la Sen ${ }^{3 *}$
}

\section{"Correspondence: \\ manuel.delasen@ehu.es \\ ${ }^{3}$ Institute for Research and \\ Development of Processes, \\ University of Basque Country, \\ Campus of Leioa (Bizkaia), Apartado \\ 644, Bilbao, Leioa 48090, Spain \\ Full list of author information is \\ available at the end of the article}

\begin{abstract}
Coincidence and common fixed point theorems for a class of Suzuki hybrid contractions involving two pairs of single-valued and multivalued maps in a metric space are obtained. In addition, the existence of a common solution for a certain class of functional equations arising in a dynamic programming is also discussed.
\end{abstract}

MSC: $47 \mathrm{H} 10 ; 54 \mathrm{H} 25$

Keywords: coincidence point; fixed point; Hausdorff metric space; Suzuki hybrid contraction; IT-commuting maps; functional equations; dynamic programming

\section{Introduction}

Consistent with [1] (see also [2]), $Y$ denotes an arbitrary nonempty set, $(X, d)$ a metric space and $C L(X)$ (resp. $C B(X)$ ), the collection of all nonempty closed (resp. closed bounded) subsets of $X$. The hyperspace $(C L(X), H)$ (resp. $(C B(X), H))$ is called the generalized Hausdorff (resp. the Hausdorff) metric space induced by the metric $d$ on $X$.

For nonempty subsets $A, B$ of $X, d(A, B)$ denotes the gap between the subsets $A$ and $B$, while

$$
\begin{aligned}
& \rho(A, B)=\sup \{d(a, b): a \in A, b \in B\}, \\
& B N(X)=\{A: \varnothing \neq A \subseteq X \text { and the diameter of } A \text { is finite }\} .
\end{aligned}
$$

As usual, we write $d(x, B)$ (resp. $\rho(x, B))$ for $d(A, B)$ (resp. $\rho(A, B)$ ) when $A=\{x\}$.

For the sake of brevity, we choose the following notations, wherein $S, T, f$, and $g$ are maps to be defined specifically in a particular context, while $x$ and $y$ are elements of some specific domain:

$$
\begin{aligned}
& M(S, T ; f x, g y)=\max \left\{d(f x, g y), d(f x, S x), d(g y, T y), \frac{d(S x, g y)+d(T y, f x)}{2}\right\} ; \\
& M(S x, T y)=\max \left\{d(x, y), d(x, S x), d(y, T y), \frac{d(S x, y)+d(T y, x)}{2}\right\} .
\end{aligned}
$$

Let $C B(X)$ denote the class of all nonempty closed bounded subsets of $X$.

A map $T: X \rightarrow C B(X)$ is called a Nadler multivalued contraction if there exists $k \in[0,1)$ such that, for every $x, y \in X, H(T x, T y) \leq k d(x, y)$.

(c) 2014 Singh et al.: licensee Springer. This is an Open Access article distributed under the terms of the Creative Commons Attribution License (http://creativecommons.org/licenses/by/2.0), which permits unrestricted use, distribution, and reproduction in any medium, provided the original work is properly cited. 
The classical multivalued contraction theorem due to Nadler [1] states that Nadler multivalued contraction on a complete metric space $X$ has a fixed point in $X$, that is, there exists $z \in X$ such that $z \in T z$. For a detailed discussion of this theorem on generalized Hausdorff metric spaces and applications, one may refer to [3-13], and [14].

Nadler's multivalued contraction theorem [1] has led to a rich fixed point theory for multivalued maps in nonlinear analysis (see, for instance [6, 9-12, 15-22], and [13, 14, 23, 24]). It has various applications in mathematical sciences (see, for instance, [2, 5, 7-9], and [25]).

The following important result involving two pairs of hybrid maps on an arbitrary nonempty set with values in a metric space is due to Singh and Mishra [12] (see also [21]).

Theorem 1.1 Let $S, T: Y \rightarrow C L(X)$ and $f, g: Y \rightarrow X$ be such that $S(Y) \subseteq g(Y)$ and $T(Y) \subseteq$ $f(Y)$ and one of $S(Y), T(Y), f(Y)$ or $g(Y)$ is a complete subspace of $X$. Assume there exists $r \in[0,1)$ such that, for every $x, y \in Y$,

$$
H(S x, T y) \leq r M(S, T ; f x, g y) .
$$

Then

(i) S and $f$ have a coincidence point $v$ in $Y$;

(ii) $T$ and $g$ have a coincidence point $w$ in $Y$.

Further, if $Y=X$, then

(iii) $S$ and $f$ have a common fixed point $v$ provided that $f v$ is a fixed point of $f$, and $f$ and $S$ commute at $v$;

(iv) $T$ and $g$ have a common fixed point $w$ provided that gw is a fixed point of $g$, and $g$ and $T$ commute at $w$;

(v) $S, T$, f, and $g$ have a common fixed point provided that (iii) and (iv) both are true.

The following result due to Kikkawa and Suzuki [26] (see also $[13,14]$ ) generalizes Nadler's multivalued contraction theorem.

Theorem 1.2 Let $X$ be a complete metric space and $T: X \rightarrow C B(X)$. Assume there exists $r \in[0,1)$ such that, for every $x, y \in X$,

$$
d(x, T x) \leq(1+r) d(x, y)
$$

implies

$$
H(T x, T y) \leq r d(x, y)
$$

Then $T$ has a fixed point in $X$.

Subsequently, some interesting extensions and generalizations of Theorem 1.2 have recently been obtained among others by Abbas et al. [27], Dhompongsa and Yingtaweesittikul [18], Doric̀ and Lazovic̀ [28], Kamal et al. [29], Moț and Petruşel [19], Singh and Mishra [13, 14] and Singh et al. [10, 30], and [23].

The importance of Suzuki contraction theorem [24, Theorem 2], Theorem 1.2 and subsequently obtained coincidence and fixed point theorems ( $c f$. [13, 14, 18, 19, 23, 26-28], 
and others) for maps in metric spaces satisfying Suzuki type contractive conditions is that the contractive conditions are required to be satisfied not for all points of the domain. For example, the condition (1.1) of Theorem 1.2 puts some restrictions on the domain of the map $T$.

In all that follows, we take a nonincreasing function $\varphi$ from $[0,1)$ onto $(0,1]$ defined by

$$
\varphi(r)= \begin{cases}1 & \text { if } 0 \leq r<\frac{1}{2} \\ 1-r & \text { if } \frac{1}{2} \leq r<1\end{cases}
$$

Recently, Singh et al. [10] obtained the following coincidence and common fixed point theorem which generalizes a result of Doric̀ and Lazovic̀ [28] and some other results from $[3,26]$, and [21].

Theorem 1.3 Let $S, T: Y \rightarrow C L(X)$ and $f: Y \rightarrow X$ be such that $S(Y) \subseteq f(Y)$ and $T(Y) \subseteq$ $f(Y)$. Assume there exists $r \in[0,1)$ such that, for every $x, y \in Y$,

$$
\varphi(r) \min \{d(f x, S x), d(f y, T y)\} \leq d(f x, f y)
$$

implies

$$
H(S x, T y) \leq r M(S x, T y ; f x, f y) .
$$

If one of $S(Y), T(Y)$ or $f(Y)$ is a complete subspace of $X$, then there exists a point $z \in Y$ such that $f z \in S z \cap T z$.

Further, if $Y=X$, and $f z$ is a fixed point off, then $f z$ is common fixed point of $S$ and $T$ provided that $f$ is IT (Itoh-Takahashi)-commuting [13] with $S$ and $T$ at $z$.

Now a natural question arises whether Theorem 1.1 can further be generalized. In this paper, we answer this question affirmatively under tight minimal conditions. Our main result (Theorem 2.2) also presents an extension of Theorem 1.3 for a quadruplet of maps. Some recent results are discussed as special cases. Further, using two corollaries of the main result (Theorem 2.2), we obtain other common fixed point theorems for multivalued and single-valued maps on metric spaces. We also deduce the existence of common solution for a certain class of functional equations arising in dynamic programming. Examples are given to justify applications.

\section{Main results}

The following definition is due to Itoh and Takahashi [31] (see also [13]).

Definition 2.1 Let $T: X \rightarrow C L(X)$ and $f: X \rightarrow X$. Then the hybrid pair $(T, f)$ is ITcommuting at $z \in X$ if $f T z \subseteq T f z$.

Evidently a pair of commuting multivalued map $T: X \rightarrow C L(X)$ and a single-valued map $f: X \rightarrow X$ are IT-commuting but the reverse implication is not true [32, p.2]. However, a pair of single-valued maps $f, g: X \rightarrow X$ are IT-commuting (also called weakly compatible by Jungck and Rhoades [33]) at $x \in X$ if $f g x=g f x$ when $f x=g x$. 
We shall need the following lemma, essentially due to Nadler [1] (see also [3], [2, p.61], $[9$, p.76].

Lemma 2.1 If $A, B \in C L(X)$ and $a \in A$, then for each $\varepsilon>0$, there exists $b \in B$ such that $d(a, b) \leq H(A, B)+\varepsilon$.

Let $C(S, f)$ denote the collection of all coincidence points of $S$ and $f$, that is, $C(S, f)=$ $\{z \in Y: f z \in S z\}$ when $S: Y \rightarrow C L(X)$ and $f: Y \rightarrow X$; and $C(S, f)=\{z \in Y: f z=S z\}$ when $S, f: Y \rightarrow X$. The following is the main result of this section.

Theorem 2.2 Let $S, T: Y \rightarrow C L(X)$ and $f, g: Y \rightarrow X$ be such that $S(Y) \subseteq g(Y)$ and $T(Y) \subseteq$ $f(Y)$. Assume there exists $r \in[0,1)$ such that, for every $x, y \in Y$,

$$
\varphi(r) \min \{d(f x, S x), d(g y, T y)\} \leq d(f x, g y)
$$

implies

$$
H(S x, T y) \leq r M(S, T ; f x, g y) .
$$

If one of $S(Y), T(Y), f(Y)$ or $g(Y)$ is a complete subspace of $X$, then

(I) $C(S, f)$ is nonempty, i.e. there exists a point $z \in Y$ such that $f z \in S z$;

(II) $C(T, g)$ is nonempty, i.e. there exists a point $z_{1} \in Y$ such that $g z_{1} \in T z_{1}$.

Furthermore, if $Y=X$, then

(III) $S$ and $f$ have a common fixed point provided that the maps $S$ and $f$ are IT-commuting just at coincidence point $z$ and $f z$ is fixed point of $f$;

(IV) $T$ and $g$ have a common fixed point provided that the maps $T$ and $g$ are IT-commuting just at coincidence point $z_{1}$ and $g z_{1}$ is fixed point of $g$;

(V) $S, T$, $f$, and $g$ have a common fixed point provided that both (III) and (IV) are true.

Proof Without loss of generality, we may take $r>0$ and $f, g$ non-constant maps.

Let $\varepsilon>0$ be such that $\beta=r+\varepsilon<1$. We construct two sequences $\left\{x_{n}\right\}$ and $\left\{y_{n}\right\}$ in $Y$ as follows.

Let $x_{0} \in Y$ and $y_{0}=g x_{1} \in S x_{0}$. By Lemma 2.1, there exists $y_{1}=f x_{2} \in T x_{1}$ such that

$$
d\left(f x_{2}, g x_{1}\right) \leq H\left(S x_{0}, T x_{1}\right)+\varepsilon M\left(S, T ; f x_{0}, g x_{1}\right) .
$$

Similarly, there exists $y_{2}=g x_{3} \in S x_{2}$ such that

$$
d\left(f x_{2}, g x_{3}\right) \leq H\left(S x_{2}, T x_{1}\right)+\varepsilon M\left(S, T ; f x_{2}, g x_{1}\right) .
$$

Continuing in this manner, we find a sequence $\left\{y_{n}\right\}$ in $Y$ such that

$$
y_{2 n}=g x_{2 n+1} \in S x_{2 n}, \quad y_{2 n+1}=f x_{2 n+2} \in T x_{2 n+1}
$$

and

$$
\begin{aligned}
& d\left(f x_{2 n}, g x_{2 n+1}\right) \leq H\left(S x_{2 n}, T x_{2 n-1}\right)+\varepsilon M\left(S, T ; f x_{2 n}, g x_{2 n-1}\right), \\
& d\left(f x_{2 n+2}, g x_{2 n+1}\right) \leq H\left(S x_{2 n}, T x_{2 n+1}\right)+\varepsilon M\left(S, T ; f x_{2 n}, g x_{2 n+1}\right) .
\end{aligned}
$$


Now, we show that, for any $n \in N$,

$$
d\left(y_{2 n}, y_{2 n-1}\right) \leq \beta d\left(y_{2 n-1}, y_{2 n-2}\right)
$$

Suppose if $d\left(g x_{2 n-1}, T x_{2 n-1}\right) \geq d\left(f x_{2 n}, S x_{2 n}\right)$, then

$$
\varphi(r) \min \left\{d\left(f x_{2 n}, S x_{2 n}\right), d\left(g x_{2 n-1}, T x_{2 n-1}\right)\right\} \leq d\left(f x_{2 n}, g x_{2 n-1}\right) .
$$

Therefore by the assumption,

$$
\begin{aligned}
d\left(f x_{2 n}, g x_{2 n+1}\right) \leq & H\left(S x_{2 n}, T x_{2 n-1}\right)+\varepsilon M\left(S, T ; f x_{2 n}, g x_{2 n-1}\right) \\
\leq & r M\left(S, T ; f x_{2 n}, g x_{2 n-1}\right)+\varepsilon M\left(S, T ; f x_{2 n}, g x_{2 n-1}\right) \\
= & \beta M\left(S, T ; f x_{2 n}, g x_{2 n-1}\right) \\
= & \beta \max \left\{d\left(f x_{2 n}, g x_{2 n-1}\right), d\left(f x_{2 n}, S x_{2 n}\right), d\left(g x_{2 n-1}, T x_{2 n-1}\right),\right. \\
& \left.\frac{d\left(g x_{2 n-1}, S x_{2 n}\right)+d\left(f x_{2 n}, T x_{2 n-1}\right)}{2}\right\} .
\end{aligned}
$$

This yields (2.1). Suppose if $d\left(f x_{2 n}, S x_{2 n}\right) \geq d\left(g x_{2 n-1}, T x_{2 n-1}\right)$, then

$$
\varphi(r) \min \left\{d\left(f x_{2 n}, S x_{2 n}\right), d\left(g x_{2 n-1}, T x_{2 n-1}\right)\right\} \leq d\left(f x_{2 n}, g x_{2 n-1}\right) .
$$

Therefore by the assumption,

$$
\begin{aligned}
d\left(f x_{2 n}, g x_{2 n+1}\right) \leq & H\left(S x_{2 n}, T x_{2 n-1}\right)+\varepsilon M\left(S, T ; f x_{2 n}, g x_{2 n-1}\right) \\
\leq & r M\left(S, T ; f x_{2 n}, g x_{2 n-1}\right)+\varepsilon M\left(S, T ; f x_{2 n}, g x_{2 n-1}\right) \\
= & \beta M\left(S, T ; f x_{2 n}, g x_{2 n-1}\right) \\
= & \beta \max \left\{d\left(f x_{2 n}, g x_{2 n-1}\right), d\left(f x_{2 n}, S x_{2 n}\right), d\left(g x_{2 n-1}, T x_{2 n-1}\right),\right. \\
& \left.\frac{d\left(g x_{2 n-1}, S x_{2 n}\right)+d\left(f x_{2 n}, T x_{2 n-1}\right)}{2}\right\} \\
\leq & \beta \max \left\{d\left(f x_{2 n}, g x_{2 n-1}\right), d\left(f x_{2 n}, g x_{2 n+1}\right)\right\},
\end{aligned}
$$

yielding (2.1). So, in both cases we obtain (2.1). In an analogous manner, we show that

$$
d\left(y_{2 n+1}, y_{2 n}\right) \leq \beta d\left(y_{2 n}, y_{2 n-1}\right)
$$

We conclude from (2.1) and (2.2) that, for any $n \in N$,

$$
d\left(y_{n+1}, y_{n}\right) \leq \beta d\left(y_{n}, y_{n-1}\right)
$$

Therefore the sequence $\left\{y_{n}\right\}$ is Cauchy. Assume that the subspace $g(Y)$ is complete. Notice that the sequence $\left\{y_{2 n}\right\}$ is contained in $g(Y)$ and has a limit in $g(Y)$. Call it $u$. Let $z \in f^{-1} u$. Then $z \in Y$ and $f z=u$. The subsequence $\left\{y_{2 n+1}\right\}$ also converges to $u$. Let $z_{1} \in g^{-1} u$. Then 
Now we show that, for any $g y \in X-\{f z\}$,

$$
d(u, T y) \leq r \max \{d(u, g y), d(g y, T y)\},
$$

and for any $f y \in X-\{g z\}$,

$$
d(u, S y) \leq r \max \{d(u, f y), d(f y, S y)\} .
$$

Since $f x_{2 n} \rightarrow f z$, there exists $n_{0} \in N$ (natural numbers) such that

$$
d\left(f x_{2 n}, f z\right) \leq \frac{1}{3} d(f z, g y) q \text { for } g y \neq f z \text { and all } n \geq n_{0} .
$$

Also $g x_{2 n+1} \rightarrow f z$, there exists $n_{1} \in N$ such that

$$
d\left(g x_{2 n+1}, f z\right) \leq \frac{1}{3} d(f z, g y) \quad \text { for } g y \neq f z \text { and all } n \geq n_{1} .
$$

Then as in [24, p.1862] (see also [28]),

$$
\begin{aligned}
\varphi(r) d\left(f x_{2 n}, S x_{2 n}\right) & \leq d\left(f x_{2 n}, S x_{2 n}\right) \leq d\left(f x_{2 n}, g x_{2 n+1}\right) \\
& \leq \frac{2}{3} d(f z, g y) \\
& =d(f z, g y)-\frac{1}{3} d(f z, g y) \\
& \leq d(f z, g y)-d\left(f x_{2 n}, f z\right) \\
& \leq d\left(f x_{2 n}, g y\right) .
\end{aligned}
$$

Therefore

$$
\varphi(r) d\left(f x_{2 n}, S x_{2 n}\right) \leq d\left(f x_{2 n}, g y\right) .
$$

Now, either $d\left(f x_{2 n}, S x_{2 n}\right) \leq d(g y, T y)$ or $d(g y, T y) \leq d\left(f x_{2 n}, S x_{2 n}\right)$.

In either case, by (2.6) and the assumption,

$$
\begin{aligned}
d\left(f x_{2 n+1}, T y\right) \leq & H\left(S x_{2 n}, T y\right) \leq r M\left(S, T ; f x_{2 n}, g y\right) \\
\leq & r \max \left\{d\left(f x_{2 n}, g y\right), d\left(f x_{2 n}, S x_{2 n}\right), d(g y, T y),\right. \\
& \left.\frac{d\left(f x_{2 n}, T y\right)+d\left(g y, S x_{2 n}\right)}{2}\right\} .
\end{aligned}
$$

Making $n \rightarrow \infty$,

$$
\begin{aligned}
d(u, T y) & \leq r \max \left\{d(u, g y), d(u, u), d(g y, T y), \frac{d(u, T y)+d(u, g y)}{2}\right\}, \\
& \leq r \max \left\{d(u, g y), d(g y, T y), \frac{d(u, T y)+d(u, g y)}{2}\right\},
\end{aligned}
$$

that is, $d(u, T y) \leq r \max \{d(u, g y), d(g y, T y)\}$. 
This yields (2.4), that is,

$$
d(f z, T y) \leq r \max \{d(f z, g y), d(g y, T y)\} .
$$

Analogously, we can prove (2.5), that is,

$$
d\left(g z_{1}, S y\right) \leq r \max \left\{d\left(g z_{1}, f y\right), d(f y, S y)\right\} .
$$

Now, we show that $C(S, f)$ is nonempty.

First we consider the case $0 \leq r<\frac{1}{2}$.

Suppose $f z \notin S z$. Then as in [18, p.6], let $g a \in S z$ be such that $2 r d(g a, f z)<d(S z, f z)$.

Since $g a \in S z$ implies $g a \neq f z$, we have from (2.4) and (2.5),

$$
d(f z, T a) \leq r \max \{d(f z, g a), d(g a, T a)\} .
$$

On the other hand, since $\varphi(r) d(f z, S z) \leq d(f z, S z) \leq d(f z, g a)$,

$$
\varphi(r) \min \{d(f z, S z), d(g a, T a)\} \leq d(f z, g a) .
$$

Therefore, by the given assumption,

$$
\begin{aligned}
d(g a, T a) & \leq H(S z, T a) \\
& \leq r \max \left\{d(f z, g a), d(f z, S z), d(g a, T a), \frac{d(f z, T a)+d(g a, S z)}{2}\right\} \\
& =r \max \{d(f z, g a), d(g a, T a)\} .
\end{aligned}
$$

This gives $d(g a, T a) \leq H(S z, T a) \leq r d(f z, g a)<d(f z, g a)$.

So by $(2.7), d(f z, T a) \leq r d(f z, g a)$.

Therefore,

$$
\begin{aligned}
d(f z, S z) & \leq d(f z, T a)+H(S z, T a) \leq r d(f z, g a)+r d(f z, g a) \\
& =2 r d(f z, g a)<d(f z, S z) .
\end{aligned}
$$

This contradicts $f z \notin S z$. Consequently $f z \in S z$, and $C(S, f)$ is nonempty.

In an analogous manner, we can prove in the case $0 \leq r<\frac{1}{2}$ that $C(T, g)$ is nonempty.

Now we consider the case $\frac{1}{2} \leq r<1$.

We first show that

$$
H(S z, T y) \leq r \max \left\{d(f z, g y), d(f z, S z), d(g y, T y), \frac{d(g y, S z)+d(f z, T y)}{2}\right\}
$$

Assume that $f z \neq g y$. Then for every $n \in N$, there exists $z_{n} \in T y$ such that

$$
d\left(f z, z_{n}\right) \leq d(f z, T y)+\frac{1}{n} d(f z, g y)
$$


Therefore

$$
\begin{aligned}
d(g y, T y) & \leq d\left(g y, z_{n}\right) \\
& \leq d(g y, f z)+d\left(f z, z_{n}\right) \\
& \leq d(g y, f z)+d(f z, T y)+\frac{1}{n} d(f z, g y) .
\end{aligned}
$$

So, using (2.5), the inequality (2.8) implies

$$
d(g y, T y) \leq d(f z, g y)+r \max \{d(f z, g y), d(g y, T y)\}+\frac{1}{n} d(f z, g y) .
$$

If $d(f z, g y) \geq d(g y, T y)$, then (2.9) gives

$$
\begin{aligned}
d(g y, T y) & \leq d(f z, g y)+r d(f z, g y)+\frac{1}{n} d(f z, g y) \\
& =\left(1+r+\frac{1}{n}\right) d(f z, g y) .
\end{aligned}
$$

Making $n \rightarrow \infty$,

$$
d(g y, T y) \leq(1+r) d(f z, g y) .
$$

Thus

$$
\varphi(r) d(g y, T y)=(1-r) d(g y, T y) \leq\left(\frac{1}{1+r}\right) d(g y, T y) \leq d(f z, g y) .
$$

Then

$$
\varphi(r) \min \{d(f z, S z), d(g y, T y)\} \leq d(f z, g y)
$$

and by the assumption,

$$
H(S z, T y) \leq r \max \left\{d(f z, g y), d(f z, S z), d(g y, T y), \frac{d(g y, S z)+d(f z, T y)}{2}\right\} .
$$

If $d(f z, g y)<d(g y, T y)$, then (2.9) gives

$$
d(g y, T y) \leq d(f z, g y)+r d(g y, T y)+\frac{1}{n} d(f z, g y)
$$

that is, $(1-r) d(g y, T y) \leq\left(1+\frac{1}{n}\right) d(f z, g y)$.

Making $n \rightarrow \infty$,

$$
\varphi(r) d(g y, T y) \leq d(f z, g y) .
$$

Then $\varphi(r) \min \{d(f z, S z), d(g y, T y)\} \leq d(f z, g y)$, and by the assumption, we get (2.10).

Now taking $y=u_{2 n+1}$ in (2.10) and passing to the limit, we obtain $d(f z, S z) \leq r d(f z, S z)$. 
This gives $f z \in S z$, that is, $z$ is a coincidence point of $f$ and $S$. Analogously, $f z \in T z$. Thus (I) and (II) are completely proved.

Further, if $Y=X$, and $f z$ is a fixed point of $f$, and $S$ and $f$ are IT-commuting at $z$, then $f S z \subseteq S f z$. Therefore, $f z \in S z$ implies $f f z \in f S z \subseteq S f z$, so $f z \in S f z$. This proves that $u=f z$ is a common fixed point of $f$ and $S$. Therefore (2.3) implies that $u$ is a common fixed point of $f$ and $S$. This proves (III). Analogously, $T$ and $g$ have a common fixed point $g z_{1}$. Therefore (2.3) implies that $u$ is a common fixed point of $T$ and $g$. This proves (IV). Now (V) is immediate.

Remark 2.1 In Theorem 2.2, the hypothesis ' $f z$ is a fixed point of $f$ ' is essential for the existence of a common fixed point of $S$ and $f$ (see [22,34] and the following example). Similarly, the hypothesis ' $g z_{1}$ is a fixed point of $g$ ' is essential for the existence of a common fixed point of $T$ and $g$.

Example 2.3 Let $X=R^{+}$(nonnegative reals) be endowed with the usual metric. Define for $x \in X, f x=2 x^{2}, g x=2 x^{3}, S x=\left[\frac{1}{4}, x^{2}+\frac{1}{4}\right]$ and $T x=\left[\frac{1}{4}, x^{3}+\frac{1}{4}\right]$. Then $S(X)=T(X)=$ $\left[\frac{1}{4}, \infty\right) \subset X=f(X)=g(X)$, and all other hypotheses of Theorem 2.2 with $Y=X=R^{+}$are satisfied for $r=\frac{1}{2}=\varphi(r)$. Notice that $g z_{1}=T z_{1}=\frac{1}{2}$, where $z_{1}=4^{-1 / 3}$. Thus $g$ and $T$ have a coincidence at $z_{1}$, but $g z_{1}=\frac{1}{2}$ is not a fixed point of $g$ and hence not a common fixed point of $g$ and $T$. Note that $z=\frac{1}{2}$ is a coincidence point of $f$ and $S$, and $S f(z)=\left[\frac{1}{8}, \frac{1}{2}\right] \subset\left[\frac{1}{4}, \frac{1}{2}\right]=f S(z)$, that is, $f$ and $S$ are IT-commuting at $z$. Evidently, $z=f(z)$ is a common fixed point of $f$ and $S$.

The following result due to Singh et al. [35] extends and generalizes certain results of $[10,12,26]$ and others.

Corollary 2.4 Let $S: Y \rightarrow C L(X)$ and $f, g: Y \rightarrow X$ be such that $S(Y) \subseteq f(Y) \cap g(Y)$. Assume there exists $r \in[0,1)$ such that, for every $x, y \in Y$,

$$
\varphi(r) \min \{d(f x, S x), d(g y, S y)\} \leq d(f x, g y)
$$

implies

$$
H(S x, S y) \leq r M(S ; f x, g y) .
$$

If one of $S(Y), f(Y)$ or $g(Y)$ is a complete subspace of $X$, then

(I) $C(S, f)$ is nonempty, i.e. there exists a point $z \in Y$ such that $f z \in S z$;

(II) $C(S, g)$ is nonempty, i.e. there exists a point $z_{1} \in Y$ such that $g z_{1} \in S z_{1}$.

Furthermore, if $Y=X$, then

(III) $S$ and $f$ have a common fixed point provided that the maps $S$ and $f$ are IT-commuting just at coincidence point $z$ and $f z$ is fixed point of $f$

(IV) $S$ and $g$ have a common fixed point provided that the maps $S$ and $g$ are IT-commuting just at coincidence point $z_{1}$ and $g z_{1}$ is fixed point of $g$;

(V) $S$, $f$, and $g$ have a common fixed point provided that both (III) and (IV) are true.

Proof It follows from Theorem 2.2 when $T=S$. 
We remark that in general the coincidence points $z$ and $z_{1}$ guaranteed by Theorem 2.2 or Corollary 2.4 may be different. However, if we take $f=g$ in Theorem 2.2, the maps $S$, $T$, and $f$ have a common coincidence point. So we have a slightly sharp result.

\section{Corollary 2.5 Theorem 1.3.}

Proof It follows from Theorem 2.2 when $g=f$.

The following result extends and generalizes certain results of $[28,36]$ and others.

Corollary 2.6 [23] Let $X$ be a complete metric space and $S, T: X \rightarrow C L(X)$. Assume there exists $r \in[0,1)$ such that, for every $x, y \in X$,

$$
\varphi(r) \min \{d(x, S x), d(y, T y)\} \leq d(x, y) \quad \text { implies } \quad H(S x, T y) \leq r M(S x, T y) .
$$

Then there exists an element $z \in X$ such that $z \in S z \cap T z$.

Proof It follows from Theorem 2.2 when $Y=X$ and $f$ and $g$ are the identity maps on $Y=X$.

The following result due to Doric̀ and Lazovic̀ [28] generalizes many fixed point theorems from $[13,26]$ and [37].

Corollary 2.7 Let $X$ be a complete metric space and $S: X \rightarrow C L(X)$. Assume there exists $r \in[0,1)$ such that, for every $x, y \in X$,

$$
\varphi(r) d(x, S x) \leq d(x, y) \quad \text { implies } \quad H(S x, S y) \leq r M(S x, S y) .
$$

Then there exists an element $z \in X$ such that $z \in S z$.

Proof It follows from Theorem 2.2 when $Y=X, T=S$, and $f, g$ are the identity maps on $X$.

The following result extends a common fixed point theorem of [10, Theorem 2.8].

Corollary 2.8 Let $f, g, P, Q: Y \rightarrow X$ be such that $P(Y) \subseteq g(Y), Q(Y) \subseteq f(Y)$, and one of $P(Y)$ or $Q(Y)$ or $f(Y)$ or $g(Y)$ is complete subspace of $X$. Assume there exists $r \in[0,1)$ such that, for every $x, y \in Y$,

$$
\varphi(r) \min \{d(f x, P x), d(g y, Q y)\} \leq d(f x, g y)
$$

implies

$$
d(P x, Q y) \leq r M(P, Q ; f x, g y) .
$$

Then $C(P, f)$ and $C(Q, g)$ are nonempty. Further, if $Y=X$, and if $f, g, P$, and $Q$ are commuting at a common coincidence point, then $f, g, P$, and $Q$ have a unique common fixed point, that is, there exists a unique point $z \in X$ such that $f z=g z=P z=Q z=z$. 
Proof Set $S x=\{P x\}$ and $T x=\{Q x\}$ for every $x \in Y$. Then it easily comes from Theorem 2.2 that $C(P, f)$ and $C(Q, g)$ are nonempty. Furthermore, if $Y=X$ and $f$ and $g$ commute, respectively, with $P$ and $Q$ at $z$, then $f f z=f P z=P f z$, $f f z=f Q z=Q f z, g g z=g P z=P g z$, and $g g z=g Q z=Q g z$.

Also $\varphi(r) \min \{d(f z, P z), d(f f z, Q f z)\}=0 \leq d(f z, f f z)$, and this implies

$$
\begin{aligned}
d(P z, Q f z) \leq & r \max \{d(f z, f f z), d(f z, P z), d(f f z, Q f z), \\
& \left.\frac{d(f z, Q f z)+d(f f z, P z)}{2}\right\} \\
= & r d(P z, Q f z) .
\end{aligned}
$$

This says that $f z$ is fixed point of $f$ and $P$. Analogously $g z$ is fixed point of $g$ and $Q$. The uniqueness of the common fixed point follows easily.

The following result extends and generalizes coincidence and common fixed point theorems of Goebel [38], Jungck [39], Fisher [40], and others.

Corollary 2.9 [35] Let $f, g, P: Y \rightarrow X$ be such that $P(Y) \subseteq f(Y) \cap g(Y)$. Let $P(Y)$ or $f(Y)$ or $g(Y)$ be a complete subspace of $X$. Assume there exists $r \in[0,1)$ such that, for every $x, y \in Y$,

$$
\varphi(r) \min \{d(f x, P x), d(g y, P y)\} \leq d(f x, g y)
$$

implies

$$
d(P x, P y) \leq r M(P ; f x, g y) .
$$

Then $C(P, f)$ and $C(P, g)$ are nonempty. Further, if $Y=X$ and if $P$ commutes with $f$ and $g$ at a common coincidence point, then $f, g$, and $P$ have a unique common fixed point, that is, there exists a unique point $z \in X$ such that $f z=g z=P z=z$.

Proof It follows from Corollary 2.8 when $Q=P$.

Corollary 2.10 Let $(X, d)$ be a complete metric space and $f, g: X \rightarrow X$ be onto maps. Assume there exists $r \in[0,1)$ such that, for every $x, y \in X$,

$$
\varphi(r) \min \{d(x, f x), d(y, g y)\} \leq d(f x, g y) \quad \text { implies } \quad d(x, y) \leq r M_{1}(f x, g y) .
$$

Then $f$ and $g$ have a unique common fixed point.

Proof It follows from Corollary 2.8 when $Y=X$ and $P, Q$ both are the identity maps on $X$.

Corollary 2.11 Let $(X, d)$ be a complete metric space and $f: X \rightarrow X$ be an onto map. Assume there exists $r \in[0,1)$ such that, for every $x, y \in X$,

$$
\varphi(r) d(x, f x) \leq d(f x, f y) \quad \text { implies } \quad d(x, y) \leq r M(f x, f y) .
$$

Then $f$ has a unique fixed point. 
Proof It follows from Corollary 2.10 when $f=g$.

The following example shows that Theorem 2.2 is indeed more general than Theorem 1.1.

Example 2.12 Consider a metric space $X=\{(0,0),(0,1),(1,0),(1,2),(2,1)\}$, where $d$ is defined by

$$
d\left[\left(x_{1}, x_{2}\right),\left(y_{1}, y_{2}\right)\right]=\left|x_{1}-y_{1}\right|+\left|x_{2}-y_{2}\right| .
$$

Let $S, T, f$ and $g: X \rightarrow X$ be such that

$$
\begin{aligned}
& S\left(x_{1}, x_{2}\right)= \begin{cases}(0,0) & \text { if }\left(x_{1}, x_{2}\right) \neq(1,2),(2,1), \\
(1,0) & \text { if }\left(x_{1}, x_{2}\right)=(1,2), \\
(0,1) & \text { if }\left(x_{1}, x_{2}\right)=(2,1),\end{cases} \\
& T\left(x_{1}, x_{2}\right)= \begin{cases}(0,0) & \text { if }\left(x_{1}, x_{2}\right) \neq(1,2),(2,1), \\
(0,1) & \text { if }\left(x_{1}, x_{2}\right)=(1,2), \\
(1,0) & \text { if }\left(x_{1}, x_{2}\right)=(2,1),\end{cases} \\
& f\left(x_{1}, x_{2}\right)= \begin{cases}\left(x_{2}, x_{1}\right) & \text { if }\left(x_{1}, x_{2}\right) \neq(1,2),(2,1), \\
\left(x_{1}, x_{2}\right) & \text { if }\left(x_{1}, x_{2}\right)=(1,2),(2,1)\end{cases}
\end{aligned}
$$

and

$$
g\left(x_{1}, x_{2}\right)=\left(x_{1}, x_{2}\right) \quad \text { for all }\left(x_{1}, x_{2}\right) \in X .
$$

Then $S, T, f$, and $g$ do not satisfy the assumption in Theorem 1.1 at $x=(1,2), y=(1,2)$ or at $x=(2,1), y=(2,1)$. However,

$$
d(S x, T y) \leq \frac{1}{2} \max \left\{d(f x, g y), d(f x, S x), d(g y, T y), \frac{d(S x, g y)+d(T y, f x)}{2}\right\}
$$

if $(x, y) \neq((1,2),(1,2))$ and $(x, y) \neq((2,1),(2,1))$.

Since at $(x, y)=((1,2),(1,2)), \varphi(r) \min \{d(f x, S x), d(g y, T y)\}=\varphi(r) \min \{d(f(1,2), S(1,2))$, $d(g(1,2), T(1,2))\}=\varphi(r) \min \{2,2\}=2 \varphi(r)$.

Here we note that the value of $r$ is $1 / 2$, so by definition, $\varphi(r)=1 / 2$, so $\varphi(r) \min \{d(f x, S x)$, $d(g y, T y)\}=1>0=d(f x, g y)$.

Thus $S, T, f$, and $g$ satisfy the assumption of Theorem 2.2 (and also Corollary 2.8).

In the following example, we show that two multivalued maps and two single-valued maps satisfy all the hypotheses of Theorem 2.2 to ensure common coincidence points of pairwise maps.

Example 2.13 Let $Y=\{a, b, c, d\}$ and $X=\{2,3,4,5,7\}$. Let $d$ be the usual metric on $X$, and $S, T, f$, and $g$ be defined on $Y$ with values in $X$ as

$$
S(x)= \begin{cases}\{2,3,4\} & \text { if } x=a, b, c \\ \{2\} & \text { if } x=d,\end{cases}
$$




$$
\begin{gathered}
T(x)= \begin{cases}\{2,3,4\} & \text { if } x=a, b, c, \\
\{3\} & \text { if } x=d,\end{cases} \\
f(x)= \begin{cases}4 & \text { if } x=a, \\
2 & \text { if } x=b, \\
3 & \text { if } x=c, \\
7 & \text { if } x=d\end{cases}
\end{gathered}
$$

and

$$
g(x)= \begin{cases}2 & \text { if } x=a, \\ 4 & \text { if } x=b \\ 3 & \text { if } x=c \\ 5 & \text { if } x=d .\end{cases}
$$

Notice that $S(Y) \subset g(Y)$ and $T(Y) \subset f(Y)$. Further, all other conditions of Theorem 2.2 are readily verified with $r=2 / 3$ and $\varphi(r)=1 / 3$. Evidently, $f a \in S a, f b \in S b, f c \in S c$, and $g a \in T a$, $g b \in T b, g c \in T c$. Moreover, $C(f, S)=C(g, T)=\{b, c, d\}$.

Now we give an application of Corollary 2.8.

Theorem 2.14 Let $S, T: Y \rightarrow B N(X)$ and $f, g: Y \rightarrow X$ be such that $S(Y) \subseteq g(Y), T(Y) \subseteq$ $f(Y)$, and let one of $S(Y), T(Y), f(Y)$ or $g(Y)$ be a complete subspace of $X$. Assume there exists $r \in[0,1)$ such that, for every $x, y \in Y$,

$$
\varphi(r) \min \{\rho(f x, S x), \rho(g y, T y)\} \leq d(f x, g y)
$$

implies

$$
\rho(S x, T y) \leq r \max \left\{d(f x, g y), \rho(f x, S x), \rho(g y, T y), \frac{d(f x, T y)+d(g y, S x)}{2}\right\} .
$$

Then $C(S, f)$ and $C(T, g)$ are nonempty.

Proof Choose $\lambda \in(0,1)$. Define single-valued maps $h_{1}, h_{2}: X \rightarrow X$ as follows. For each $x \in X$, let $h_{1} x$ be a point of $S x$ which satisfies

$$
d\left(f x, h_{1} x\right) \geq r^{\lambda} \rho(f x, S x) .
$$

Similarly, for each $y \in X$, let $h_{2} y$ be a point of $T y$ such that

$$
d\left(g y, h_{2} y\right) \geq r^{\lambda} \rho(g y, T y) .
$$

Since $h_{1} x \in S x$ and $h_{2} y \in T y$,

$$
d\left(f x, h_{1} x\right) \leq \rho(f x, S x) \quad \text { and } \quad d\left(g y, h_{2} y\right) \leq \rho(g y, T y) .
$$


So (2.11) gives

$$
\varphi(r) \min \left\{d\left(f x, h_{1} x\right), d\left(g y, h_{2} y\right)\right\} \leq \varphi(r) \min \{\rho(f x, S x), \rho(g y, T y)\} \leq d(f x, g y),
$$

and this implies (2.12). Therefore

$$
\begin{aligned}
d\left(h_{1} x, h_{2} y\right) \leq & \rho(S x, T y) \\
\leq & r \cdot r^{-\lambda} \max \left\{r^{\lambda} d(f x, g y), r^{\lambda} \rho(f x, S x), r^{\lambda} \rho(g y, T y),\right. \\
& \left.\frac{r^{\lambda} d(f x, T y)+r^{\lambda} d(g y, S x)}{2}\right\} \\
\leq & r^{1-\lambda} \max \left\{d(f x, g y), d\left(f x, h_{1} x\right), d\left(g y, h_{2} y\right),\right. \\
& \left.\frac{d\left(f x, h_{2} y\right)+d\left(g y, h_{1} x\right)}{2}\right\} .
\end{aligned}
$$

So (2.13), viz., $\varphi\left(r^{\prime}\right) \min \left\{d\left(f x, h_{1} x\right), d\left(g y, h_{2} y\right)\right\} \leq d(f x, g y)$ implies

$$
\begin{aligned}
d\left(h_{1} x, h_{2} y\right) \leq & r^{\prime} \max \left\{d(f x, g y), d\left(f x, h_{1} x\right), d\left(g y, h_{2} y\right),\right. \\
& \left.\frac{d\left(f x, h_{2} y\right)+d\left(g y, h_{1} x\right)}{2}\right\}
\end{aligned}
$$

where $r^{\prime}=r^{1-\lambda}<1$.

Hence by Corollary 2.8, there exist $z_{1}, z_{2} \in Y$ such that $h_{1} z_{1}=f z_{1}$ and $h_{2} z_{2}=g z_{2}$. This implies that $z_{1}$ is a coincidence point of $f$ and $S$, and $z_{2}$ is a coincidence point of $g$ and $T$.

Corollary 2.15 Let $S: Y \rightarrow B N(X)$ and $f, g: Y \rightarrow X$ be such that $S(Y) \subseteq f(Y) \cap g(Y)$, and let one of $S(Y), f(Y)$ or $g(Y)$ be a complete subspace of $X$. Assume there exists $r \in[0,1)$ such that, for every $x, y \in Y$,

$$
\varphi(r) \min \{\rho(f x, S x), \rho(g y, S y)\} \leq d(f x, g y)
$$

implies

$$
\rho(S x, S y) \leq r \max \left\{d(f x, g y), \rho(f x, S x), \rho(g y, S y), \frac{d(f x, S y)+d(g y, S x)}{2}\right\} .
$$

Then $C(S, f)$ and $C(S, g)$ are nonempty.

Proof It follows from Theorem 2.14 when $T=S$.

Corollary 2.16 [10] Let $S, T: Y \rightarrow B N(X)$ and $f: Y \rightarrow X$ be such that $S(Y) \subseteq f(Y), T(Y) \subseteq$ $f(Y)$ and let $S(Y)$ or $T(Y)$ or $f(Y)$ be a complete subspace of $X$. Assume there exists $r \in[0,1)$ such that, for every $x, y \in X$,

$$
\varphi(r) \min \{\rho(f x, S x), \rho(f y, T y)\} \leq d(f x, f y)
$$


implies

$$
\rho(S x, T y) \leq r \max \left\{d(f x, f y), \rho(f x, S x), \rho(f y, T y), \frac{d(f x, T y)+d(f y, S x)}{2}\right\} .
$$

Then there exists $z \in Y$ such that $f z \in S z \cap T z$.

Proof It follows from Theorem 2.14 when $g=f$.

Corollary 2.17 [23] Let $X$ be a complete metric space and let $S, T: X \rightarrow B N(X)$. Assume there exists $r \in[0,1)$ such that, for every $x, y \in X$,

$$
\varphi(r) \min \{\rho(x, S x), \rho(y, T y)\} \leq d(x, y)
$$

implies

$$
\rho(S x, T y) \leq r \max \left\{d(x, y), \rho(x, S x), \rho(y, T y), \frac{d(x, T y)+d(y, S x)}{2}\right\} .
$$

Then there exists a unique point $z \in X$ such that $z \in S z \cap T z$.

Proof It follows from Theorem 2.14 when $f$ and $g$ are the identity maps on $X$.

Corollary 2.18 Let $S: Y \rightarrow B N(X)$ and $f: Y \rightarrow X$ be such that $S(Y) \subseteq f(Y)$, and let $S(Y)$ or $f(Y)$ be a complete subspace of $X$. Assume there exists $r \in[0,1)$ such that, for every $x, y \in Y$,

$$
\varphi(r) \rho(f x, S x) \leq d(f x, f y)
$$

implies

$$
\rho(S x, S y) \leq r \max \left\{d(f x, f y), \rho(f x, S x), \rho(f y, S y), \frac{d(f x, S y)+d(f y, S x)}{2}\right\} .
$$

Then there exists $z \in Y$ such that $f z \in S z$.

Proof It follows from Theorem 2.14 when $g=f$ and $T=S$.

Corollary 2.19 Let $X$ be a complete metric space and let $S: X \rightarrow B N(X)$. Assume there exists $r \in[0,1)$ such that, for every $x, y \in X$,

$$
\varphi(r) \rho(x, S x) \leq d(x, y)
$$

implies

$$
\rho(S x, S y) \leq r \max \left\{d(x, y), \rho(x, S x), \rho(y, S y), \frac{d(x, S y)+d(y, S x)}{2}\right\} .
$$

Then there exists a unique point $z \in X$ such that $z \in S z$.

Proof It follows from Theorem 2.14 that $S$ has a fixed point when $f=g$ is the identity map on $X$ and $T=S$. The uniqueness of the fixed point follows easily. 


\section{Applications}

Throughout this section, we assume that $U$ and $V$ are Banach spaces, $W \subseteq U$ and $D \subseteq V$. Let $R$ denote the field of reals, $\tau: W \times D \rightarrow W, g, g^{\prime}: W \times D \rightarrow R$ and $G_{1}, G_{2}, F_{1}, F_{2}$ : $W \times D \times R \rightarrow R$. Considering $W$ and $D$ as the state and decision spaces respectively, the problem of dynamic programming reduces to the problem of solving the functional equations:

$$
\begin{array}{ll}
p_{i}=\sup _{y \in D}\left\{g(x, y)+G_{i}(x, y, p(\tau(x, y)))\right\}, & x \in W, i=1,2, \\
q_{i}=\sup _{y \in D}\left\{g^{\prime}(x, y)+F_{i}(x, y, q(\tau(x, y)))\right\}, & x \in W, i=1,2 .
\end{array}
$$

Indeed, in the multistage process, some functional equations arise in a natural way ( $c f$. Bellman [41] and Bellman and Lee [42]; see also [10, 43-47], and [23]). In this section, we study the existence of a common solution of the functional equations (3.1a) and (3.1b) arising in dynamic programming.

Let $B(W)$ denote the set of all bounded real-valued functions on $W$. For an arbitrary $h \in B(W)$, define $\|h\|=\sup _{x \in W}|h(x)|$. Then $(B(W),\|\cdot\|)$ is a Banach space. Suppose that the following conditions hold:

(DP-1) $G_{1}, G_{2}, F_{1}, F_{2}, g$, and $g^{\prime}$ are bounded.

(DP-2) Let $\varphi(r)$ be defined as in the previous sections. Assume that there exists $r \in[0,1)$ such that, for every $(x, y) \in W \times D, h, k \in B(W)$, and $t \in W$,

$$
\varphi(r) \min \left\{\left|J_{1} h(t)-A_{1} h(t)\right|,\left|J_{2} k(t)-A_{2} k(t)\right|\right\} \leq\left|J_{1} h(t)-J_{2} k(t)\right|
$$

implies

$$
\left|G_{1}(x, y, h(t))-G_{2}(x, y, k(t))\right| \leq r M\left(A_{1}, A_{2} ; J_{1} h, J_{2} k\right),
$$

where

$$
\begin{aligned}
M\left(A_{1}, A_{2} ; J_{1} h, J_{2} k\right) & \\
= & \max \left\{\left|J_{1} h(t)-J_{2} k(t)\right|,\left|J_{1} h(t)-A_{1} h(t)\right|,\left|J_{2} k(t)-A_{2} k(t)\right|,\right. \\
& \left.\frac{\left|J_{1} h(t)-A_{2} k(t)\right|+\left|J_{2} k(t)-A_{1} h(t)\right|}{2}\right\},
\end{aligned}
$$

and $A_{1}, A_{2}, J_{1}$, and $J_{2}$ are defined as follows:

$$
\begin{aligned}
& A_{i} h(x)=\sup _{y \in D}\left\{g(x, y)+G_{i}(x, y, h(\tau(x, y)))\right\}, \quad x \in W, h \in B(W), i=1,2, \\
& J_{i} h(x)=q=\sup _{y \in D}\left\{g^{\prime}(x, y)+F_{i}(x, y, h(\tau(x, y)))\right\}, \quad x \in W, h \in B(W), i=1,2 .
\end{aligned}
$$

(DP-3) For any $h, k \in B(W)$, there exist $u, v \in B(W)$ such that

$$
A_{1} h(x)=J_{1} u(x) \text { and } A_{2} k(x)=J_{2} v(x), \quad x \in W
$$


(DP-4) There exist $h, k \in B(W)$ such that

$$
J_{1} h(x)=A_{1} h(x) \quad \text { implies } \quad J_{1} A_{1} h(x)=A_{1} J_{1} h(x)
$$

and

$$
J_{2} k(x)=A_{2} k(x) \text { implies } J_{2} A_{2} k(x)=A_{2} J_{2} k(x) .
$$

Theorem 3.1 Assume the conditions (DP-1)-(DP-4) hold. Let $J(B(W))$ be a closed convex subspace of $B(W)$. Then the functional equations (3.1a) and (3.1b), $i=1,2$, have a unique bounded common solution in $B(W)$.

Proof For any $h, k \in B(W)$, let $d(h, k)=\sup \{|h(x)-k(x)|: x \in W\}$. Then $(B(W), d)$ is a complete metric space.

Let $\lambda$ be an arbitrary positive number and $h_{1}, h_{2} \in B(W)$. Pick $x \in W$, and choose $y_{1}, y_{2} \in$ $D$ such that

$$
A_{j} h_{j}<g\left(x, y_{j}\right)+G_{j}\left(x, y_{j}, h_{j}\left(x_{j}\right)\right)+\lambda, \quad x_{i}=\left(x, y_{i}\right), \quad i=1,2,
$$

where $x_{j}=\tau\left(x, y_{j}\right)$.

Further,

$$
\begin{aligned}
& A_{1} h_{1} \geq g\left(x, y_{2}\right)+G_{1}\left(x, y_{2}, h_{1}\left(x_{2}\right)\right), \\
& A_{2} h_{2} \geq g\left(x, y_{1}\right)+G_{2}\left(x, y_{1}, h_{2}\left(x_{1}\right)\right) .
\end{aligned}
$$

Therefore, the first inequality in (DP-2) becomes

$$
\begin{aligned}
& \varphi(r) \min \left\{\left|J_{1} h_{1}(x)-A_{1} h_{1}(x)\right|,\left|J_{2} h_{2}(x)-A_{2} h_{2}(x)\right|\right\} \\
& \quad \leq\left|J_{1} h_{1}(x)-J_{2} h_{2}(x)\right|,
\end{aligned}
$$

and this together with (3.1), (3.3), and (3.4) implies

$$
\begin{aligned}
A_{1} h_{1}-A_{2} h_{2} & <G_{1}\left(x, y_{1}, h_{1}\left(x_{1}\right)\right)-G_{2}\left(x, y, h_{2}\left(x_{1}\right)\right)+\lambda \\
& \leq\left|G_{1}\left(x, y_{1}, h_{1}\left(x_{1}\right)\right)-G_{2}\left(x, y_{1}, h_{2}\left(x_{1}\right)\right)\right|+\lambda \\
& \leq r M\left(A_{1}, A_{2} ; J_{1} h_{1}, J_{2} h_{2}\right)+\lambda .
\end{aligned}
$$

Similarly, (3.1), (3.2), and (3.4) imply

$$
A_{2} h_{2}(x)-A_{1} h_{1}(x) \leq r M\left(A_{1}, A_{2} ; J_{1} h_{1}, J_{2} h_{2}\right)+\lambda \text {. }
$$

So, from (3.5) and (3.6), we obtain

$$
\left|A_{1} h_{1}(x)-A_{2} h_{2}(x)\right| \leq r M\left(A_{1}, A_{2} ; J_{1} h_{1}, J_{2} h_{2}\right)+\lambda .
$$


As $\lambda>0$ is arbitrary and (3.7) is true for any $x \in W$, taking supremum, we find from (3.4) and (3.7) that

$$
\varphi(r) \min \left\{d\left(J_{1} h_{1}, A_{1} h_{1}\right), d\left(J_{2} h_{2}, A_{2} h_{2}\right)\right\} \leq d\left(J_{1} h_{1}, J_{2} h_{2}\right)
$$

implies

$$
d\left(A_{1} h_{1}, A_{2} h_{2}\right) \leq r M\left(A_{1}, A_{2} ; J_{1} h_{1}, J_{2} h_{2}\right) .
$$

Therefore, Corollary 2.8 applies, wherein $A_{1}, A_{2}, J_{1}$, and $J_{2}$ correspond, respectively, to the maps $P, Q, f$, and $g$. So $A_{1}, A_{2}, J_{1}$, and $J_{2}$ have a unique common fixed point $h^{*}$, that is, $h^{*}(x)$ is the unique bounded common solution of the functional equations (3.1a) and (3.1b), $i=1,2$.

Now we furnish an example in support of Theorem 3.1.

Example 3.2 Let $X=Y=R$ be a Banach space endowed with the standard norm $\|\cdot\|$ defined by $\|x\|=|x|$, for all $x \in X$. Suppose $W=[0,1] \subset X$ be the state space, and $D=$ $[0, \infty) \subset Y$ be the decision space.

Define $\tau: W \times D \rightarrow W$ by

$$
\tau(x, y)=\frac{x}{y^{2}+1}, \quad x \in W, y \in D .
$$

For any $h, k \in B(W)$, and $i=1,2$, define $p_{i}, q_{i}: W \rightarrow R$ by

$$
p_{i}(x)=q_{i}(x)=x^{2}+\frac{1}{2} .
$$

Define $G_{i}, F: W \times D \times R \rightarrow R$ by

$$
\begin{aligned}
& G_{1}(x, y, t)=\frac{1}{4}\left\{\frac{x}{(x+1)(y+1)} \sin \frac{y}{y+1}+2\right\} \\
& G_{2}(x, y, t)=\frac{1}{4}\left\{\frac{x}{(x+1)(2 y+1)} \sin \frac{y}{y+1}+2\right\} \\
& F_{1}(x, y, t)=\frac{1}{2 x+y+1}+\frac{1}{2} \sin t \\
& F_{2}(x, y, t)=\frac{1}{2 x+3 y+1}+\frac{1}{2} \sin t \\
& g(x, y)=\frac{x^{2} y^{2}}{x+y^{2}} \text { and } g^{\prime}(x, y)=\frac{x^{2} y^{5}}{x+y^{5}} .
\end{aligned}
$$

Notice that $G_{1}, G_{2}, F_{1}, F_{2}, g$, and $g^{\prime}$ are bounded. Also

$$
\begin{aligned}
& J_{1} h(x)=\sup _{y \in D}\left\{g^{\prime}(x, y)+F_{1}(x, y, h(\tau(x, y)))\right\}=x^{2}+\frac{1}{2}=q_{1}(x), \quad x \in W, h \in B(W) ; \\
& J_{2} k(x)=\sup _{y \in D}\left\{g^{\prime}(x, y)+F_{2}(x, y, k(\tau(x, y)))\right\}=x^{2}+\frac{1}{2}=q_{2}(x), \quad x \in W, h \in B(W) ;
\end{aligned}
$$




$$
\begin{array}{ll}
A_{1} h(x)=\sup _{y \in D}\left\{g(x, y)+G_{1}(x, y, h(\tau(x, y)))\right\}=x^{2}+\frac{1}{2}=p_{1}(x), \quad x \in W, h \in B(W) ; \\
A_{2} k(x)=\sup _{y \in D}\left\{g(x, y)+G_{2}(x, y, k(\tau(x, y)))\right\}=x^{2}+\frac{1}{2}=p_{2}(x), \quad x \in W, h \in B(W) .
\end{array}
$$

We see that

$$
\begin{aligned}
& \varphi(r) \min \left\{\left|J_{1} h(t)-A_{1} h(t)\right|,\left|J_{2} k(t)-A_{2} k(t)\right|\right\} \\
& \quad=\varphi(r) \min \left\{\left|q_{1}(x)-p_{1}(x)\right|,\left|q_{2}(x)-p_{2}(x)\right|\right\} \\
& \quad=0=\left|J_{1} h(t)-J_{2} k(t)\right| .
\end{aligned}
$$

Thus

$$
\varphi(r) \min \left\{\left|J h(t)-A_{1} h(t)\right|,\left|J k(t)-A_{2} k(t)\right|\right\}=|J h(t)-J k(t)|,
$$

and this implies

$$
\left|G_{1}(x, y, h(t))-G_{2}(x, y, k(t))\right|=0 \leq r M\left(A_{1}, A_{2} ; J h(t), J k(t)\right) .
$$

Finally for any $h, k \in B(W)$ with $A_{1} h=J h$, we have $A_{1} J h=p_{1}(x)=q(x)=J J h=J A_{1} h$, that is, $J A_{1} h=A_{1} J h$, and with $A_{2} k=J k$, we have $A_{2} J k=p_{2}(x)=q(x)=J J k=J A_{2} k$, that is, $J A_{2} k=$ $A_{2} J k$.

Thus all the assumptions of Theorem 3.1 are satisfied. So the system of equations (3.1a) and (3.1b) has a unique solution in $B(W)$.

Corollary 3.3 Suppose that the following conditions hold:

(i) $G, F_{1}, F_{2}, g$, and $g^{\prime}$ are bounded.

(ii) Let $\varphi(r)$ be defined as in the previous sections. Assume that there exists $r \in[0,1)$ such that, for every $(x, y) \in W \times D, h, k \in B(W)$, and $t \in W$,

$$
\varphi(r) \min \left\{\left|J_{1} h(t)-A h(t)\right|,\left|J_{2} k(t)-A k(t)\right|\right\} \leq\left|J_{1} h(t)-J_{2} k(t)\right|
$$

implies

$$
|G(x, y, h(t))-G(x, y, k(t))| \leq r M\left(A ; J_{1} h, J_{2} k\right)
$$

where

$$
\begin{aligned}
M\left(A ; J_{1} h, J_{2} k\right)= & \max \left\{\left|J_{1} h(t)-J_{2} k(t)\right|,\left|J_{1} h(t)-A h(t)\right|,\left|J_{2} k(t)-A k(t)\right|,\right. \\
& \left.\frac{\left|J_{1} h(t)-A k(t)\right|+\left|J_{2} k(t)-A h(t)\right|}{2}\right\},
\end{aligned}
$$

and $A, J_{1}$, and $J_{2}$ are defined as follows:

$$
\begin{aligned}
& A h(x)=\sup _{y \in D}\{g(x, y)+G(x, y, h(\tau(x, y)))\}, \quad x \in W, h \in B(W), \\
& J_{i} h(x)=q=\sup _{y \in D}\left\{g^{\prime}(x, y)+F_{i}(x, y, h(\tau(x, y)))\right\}, \quad x \in W, h \in B(W), i=1,2 .
\end{aligned}
$$


(iii) For any $h, k \in B(W)$, there exist $u, v \in B(W)$ such that

$$
A h(x)=J_{1} u(x) \text { and } A k(x)=J_{2} v(x), \quad x \in W .
$$

(iv) There exist $h, k \in B(W)$ such that

$$
J_{1} h(x)=A h(x) \quad \text { implies } \quad J_{1} A h(x)=A J_{1} h(x)
$$

and

$$
J_{2} k(x)=A k(x) \quad \text { implies } \quad J_{2} A k(x)=A J_{2} k(x) .
$$

Then the functional equations (3.1a) and (3.1b), $i=1,2$, have a unique bounded common solution in $B(W)$.

Proof It follows from Theorem 3.1 when $G_{1}=G_{2}=G$.

Corollary 3.4 [10] Suppose that the following conditions hold:

(i) $G_{1}, G_{2}, F, g$, and $g^{\prime}$ are bounded.

(ii) Assume there exists $r \in[0,1)$ such that, for every $(x, y) \in W \times D, h, k \in B(W)$ and $t \in W$,

$$
\varphi(r) \min \left\{\left|J h(t)-A_{1} h(t)\right|,\left|J k(t)-A_{2} k(t)\right|\right\} \leq|J h(t)-J k(t)|
$$

implies

$$
\begin{aligned}
& \left|G_{1}(x, y, h(t))-G_{2}(x, y, k(t))\right| \\
& \leq r \max \left\{|J h(t)-J k(t)|,\left|J h(t)-A_{1} h(t)\right|,\left|J k(t)-A_{2} k(t)\right|,\right. \\
& \left.\quad \frac{\left|J h(t)-A_{2} k(t)\right|+\left|J k(t)-A_{1} h(t)\right|}{2}\right\}
\end{aligned}
$$

where $A_{1}, A_{2}$, and $J$ are defined as follows:

$$
\begin{aligned}
& A_{i} h(x)=\sup _{y \in D}\left\{g(x, y)+G_{i}(x, y, h(\tau(x, y)))\right\}, \quad x \in W, h \in B(W), i=1,2, \\
& J h(x)=q=\sup _{y \in D}\left\{g^{\prime}(x, y)+F(x, y, h(\tau(x, y)))\right\}, \quad x \in W, h \in B(W) .
\end{aligned}
$$

(iii) For any $h, k \in B(W)$, there exist $u, v \in B(W)$ such that

$$
A_{1} h(x)=J u(x) \quad \text { and } \quad A_{2} k(x)=J v(x), \quad x \in W .
$$

(iv) There exist $h, k \in B(W)$ such that

$$
J h(x)=A_{1} h(x) \quad \text { implies } \quad J A_{1} h(x)=A_{1} J h(x)
$$


and

$$
J k(x)=A_{2} k(x) \quad \text { implies } J A_{2} k(x)=A_{2} J k(x) .
$$

Then the functional equations (3.1a) and (3.1b) with $F_{1}=F_{2}=F$ possesses a unique bounded common solution in $W$.

Proof It follows from Theorem 3.1 when $F_{1}=F_{2}=F$.

As an immediate consequence of Theorem 3.1 and Corollary 2.6, we obtain the following.

Corollary 3.5 [23] Suppose that the following conditions hold:

(i) $G_{1}, G_{2}$, and g are bounded.

(ii) There exists $r \in[0,1)$ such that, for every $(x, y) \in W \times D, h, k \in B(W)$, and $t \in W$,

$$
\varphi(r) \min \left\{\left|h(t)-A_{1} h(t)\right|,\left|k(t)-A_{2} k(t)\right|\right\} \leq|h(t)-k(t)|
$$

implies

$$
\begin{aligned}
& \left|G_{1}(x, y, h(t))-G_{2}(x, y, k(t))\right| \\
& \leq r \max \left\{|h(t)-k(t)|,\left|h(t)-A_{1} h(t)\right|,\left|k(t)-A_{2} k(t)\right|,\right. \\
& \left.\quad \frac{\left|h(t)-A_{2} k(t)\right|+\left|k(t)-A_{1} h(t)\right|}{2}\right\},
\end{aligned}
$$

where $A_{1}$ and $A_{2}$ are defined as follows:

$$
A_{i} h(x)=\sup _{y \in D}\left\{g(x, y)+G_{i}(x, y, h(\tau(x, y)))\right\}, \quad x \in W, h \in B(W), i=1,2 .
$$

Then the functional equation (3.1a) possesses a unique bounded solution in W.

Proof It follows from Corollary 3.4 when $g=0, \tau(x, y)=x$, and $F(x, y, t)=t$ as the assumption (DP-3) becomes redundant in this context.

The following result generalizes a recent result of Singh and Mishra [11, Corollary 4.2], which in turn extends certain results from [42] and [43].

Corollary 3.6 Suppose that the following conditions hold:

(i) G and g are bounded.

(ii) There exists $r \in[0,1)$ such that, for every $(x, y) \in W \times D, h, k \in B(W)$, and $t \in W$,

$$
\varphi(r)|h(t)-K h(t)| \leq|h(t)-k(t)|
$$

implies

$$
|G(x, y, h(t))-G(x, y, k(t))| \leq r \max M(K, K ; h(t), k(t)),
$$


where $K$ is defined as

$$
A h(x)=\sup _{y \in D}\{g(x, y)+G(x, y, h(\tau(x, y)))\}, \quad x \in W, h \in B(W) .
$$

Then the functional equation (3.1a) with $G_{1}=G_{2}=G$ possesses a unique bounded solution in $W$.

Proof It follows from Corollary 3.5 when $G_{1}=G_{2}=G$.

\section{Competing interests}

The authors declare that they have no competing interests.

\section{Authors' contributions}

All authors contributed equally to the writing of this paper. All authors read and approved the final manuscript.

\section{Author details}

${ }^{1}$ Gurukula K. Vishwavidyalaya, Haridwar, 249404, India. ${ }^{2}$ Department of Mathematics, Govt. Postgraduate College, Kanwali, Rewari, 123411, India. ${ }^{3}$ Institute for Research and Development of Processes, University of Basque Country, Campus of Leioa (Bizkaia), Apartado 644, Bilbao, Leioa 48090, Spain.

\section{Acknowledgements}

The authors thank the referees for their deep understanding, appreciation, and suggestions to improve upon the original typescript. They are also thankful to the Spanish Government for its support of this research through Grant DPI2012-30651, and to the Basque Government for its support of this research trough Grants IT378-10 and SAIOTEK S-PE12UN015. Further, they acknowledge the financial support by the University of Basque Country through Grant UFI 2011/07.

Received: 11 December 2013 Accepted: 25 June 2014 Published: 22 July 2014

\section{References}

1. Nadler, SB Jr.: Multivalued contraction mappings. Pac. J. Math. 30, 475-488 (1969)

2. Khamsi, MA, Kirk, WA: An Introduction to Metric Spaces and Fixed Point Theory. Wiley, New York (2001)

3. Cirič, LB: Fixed points for generalized multivalued contractions. Mat. Vesn. 9(24), 265-272 (1972)

4. Covitz, H, Nadler, SB Jr.: Multivalued contraction mappings in generalized metric spaces. Isr. J. Math. 8, 5-11 (1970)

5. Czerwik, S: Fixed Point Theorems and Special Solutions of Functional Equations. Scientific Publications of the University of Silesia, vol. 428. Silesian University, Katowice (1980)

6. Hadzic̀, O: A coincidence theorem for multivalued mappings in metric spaces. Stud. Univ. Babeş-Bolyai, Math. 26(4), 65-67 (1981)

7. Nadler, SB Jr.: Hyperspaces of Sets. Dekker, New York (1978)

8. Petruşel, A, Rus, IA: The theory of a metric fixed point theorem for multivalued operators. In: Proc. Ninth International Conference on Fixed Point Theory and Its Applications, Changhua, Taiwan, 16-22 July 2009, pp. 161-175. Yokohama Publ., Yokohama (2011)

9. Rus, IA: Generalized Contractions and Applications. Cluj University Press, Cluj-Napoca (2001)

10. Singh, SL, Chugh, R, Kamal, R: Suzuki type hybrid contractions and applications. Indian J. Math. 56(1), 49-76 (2014)

11. Singh, SL, Mishra, SN: Coincidence points, hybrid fixed and stationary points of orbitally weakly dissipative maps. Math. Jpn. 39, 451-459 (1994)

12. Singh, SL, Mishra, SN: On general hybrid contractions. J. Aust. Math. Soc. A 66, 244-254 (1999)

13. Singh, SL, Mishra, SN: Coincidence theorems for certain classes of hybrid contractions. Fixed Point Theory Appl. 2010, Article ID 898109 (2010)

14. Singh, SL, Mishra, SN: Fixed point theorems for single-valued and multi-valued maps. Nonlinear Anal. 74, 2243-2248 (2011)

15. Baillon, JB, Singh, SL: Nonlinear hybrid contractions on product spaces. Far East J. Math. Sci. 1, 117-127 (1993)

16. Baskaran, R, Subrahmanyam, PV: Common coincidence and fixed points. J. Math. Phys. Sci. 18, 329-343 (1984)

17. Beg, I, Azam, A: Common fixed points for commuting and compatible maps. Discuss. Math., Differ. Incl. 16, 121-135 (1996)

18. Dhompongsa, S, Yingtaweesittikul, H: Fixed points for multivalued mappings and the metric completeness. Fixed Point Theory Appl. 2009, Article ID 972395 (2009)

19. Moţ, G, Petruşel, A: Fixed point theory for a new type of contractive multi-valued operators. Nonlinear Anal. 70(9)، 3371-3377 (2009)

20. Reich, S: Fixed points of multi-valued functions. Atti Accad. Naz. Lincei, Rend. Cl. Sci. Fis. Mat. Nat. 8(51), 32-35 (1971)

21. Singh, SL, Mishra, SN: Nonlinear hybrid contractions. J. Natur. Phys. Sci. 5-8, 191-206 (1991/94)

22. Singh, SL, Mishra, SN: Coincidence and fixed points of nonself hybrid contractions. J. Math. Anal. Appl. 256(2), 486-497 (2001)

23. Singh, SL, Mishra, SN, Chugh, R, Kamal, R: General common fixed point theorems and applications. J. Appl. Math. 2012, Article ID 902312 (2012) 
24. Suzuki, T: A generalized Banach contraction principle that characterizes metric completeness. Proc. Am. Math. Soc. 136(5), 1861-1869 (2008)

25. Wegrzyk, R: Fixed point theorems for multivalued functions and their applications to functional equations. Diss. Math. 201, 1-28 (1982)

26. Kikkawa, M, Suzuki, T: Three fixed point theorems for generalized contractions with constants in complete metric spaces. Nonlinear Anal. 69(9), 2942-2949 (2008)

27. Abbas, M, Ali, B, Mishra, SN: Fixed points of multivalued Suzuki-Zamfirescu- $(f ; g)$ contraction mappings. Mat. Vesn. 66(1), 58-72 (2014)

28. Dorič, D, Lazovič, R: Some Suzuki-type fixed point theorems for generalized multivalued mappings and applications. Fixed Point Theory Appl. 2011, 40 (2011)

29. Kamal, R, Chugh, R, Singh, SL, Mishra, SN: New common fixed point theorems for multivalued maps. Appl. Gen. Topol. (2013)

30. Singh, SL, Chugh, R, Kamal, R: Suzuki type common fixed point theorems and applications. Fixed Point Theory 14(2), 1-9 (2013)

31. Itoh, S, Takahashi, W: Single-valued mappings, multivalued mappings and fixed point theorems. J. Math. Anal. Appl. 59(3), 514-521 (1977)

32. Singh, SL, Hashim, AM: New coincidence and fixed point theorems for strictly contractive hybrid maps. Aust. J. Math. Anal. Appl. 2(1), 1-7 (2005)

33. Jungck, G, Rhoades, BE: Fixed points for set-valued functions without continuity. Indian J. Pure Appl. Math. 29(3), 227-238 (1988)

34. Naimpally, SA, Singh, SL, Whitfield, JHM: Coincidence theorems for hybrid contractions. Math. Nachr. 127, 177-180 (1986)

35. Singh, SL, Kamal, R, De la Sen, M, Chugh, R: A new type of coincidence and common fixed point theorem with applications. Abstr. Appl. Anal. 2014, Article ID 642378 (2014)

36. Cirič, LB: On a family of contractive maps and fixed points. Publ. Inst. Math. (Belgr.) 17(31), $45-51$ (1974)

37. Damjanovič, B, Dorič, D: Multivalued generalizations of the Kannan fixed point theorem. Filomat 25(1), 125-131 (2011)

38. Goebel, K: A coincidence theorem. Bull. Acad. Pol. Sci., Sér. Sci. Math. Astron. Phys. 16, 733-735 (1968)

39. Jungck, G: Commuting mappings and fixed points. Am. Math. Mon. 83(4), 261-263 (1976)

40. Fisher, B: Mappings with a common fixed point. Math. Semin. Notes Kobe Univ. 7, 81-84 (1979)

41. Bellman, R: Methods of Nonlinear Analysis, vol. Il. Academic Press, New York (1973)

42. Bellman, R, Lee, ES: Functional equations in dynamic programming. Aequ. Math. 17(1), 1-18 (1978)

43. Bhakta, PC, Mitra, S: Some existence theorems for functional equations arising in dynamic programming. J. Math. Anal. Appl. 98(2), 348-362 (1984)

44. Pathak, HK, Cho, YJ, Kang, SM, Lee, BS: Fixed point theorems for compatible mappings of type (P) and applications to dynamic programming. Matematiche 50(1), 15-33 (1995)

45. Pathak, HK, Deepmala: Some existing theorems for solvability of certain function equations arising in dynamic programming. Bull. Calcutta Math. Soc. 104(3), 237-244 (2012)

46. Pathak, HK, Tiwari, R: Common fixed points for weakly compatible mappings and applications in dynamic programming. Ital. J. Pure Appl. Math. 30, 253-268 (2013)

47. Singh, SL, Mishra, SN: On a Ljubomir Ċiric fixed point theorem for nonexpansive type maps with applications. Indian J. Pure Appl. Math. 33(4), 531-542 (2002)

doi:10.1186/1687-1812-2014-147

Cite this article as: Singh et al.: Coincidence and common fixed point theorems for Suzuki type hybrid contractions

and applications. Fixed Point Theory and Applications 2014 2014:147.

\section{Submit your manuscript to a SpringerOpen ${ }^{\circ}$ journal and benefit from:}

- Convenient online submission

Rigorous peer review

- Immediate publication on acceptance

- Open access: articles freely available online

- High visibility within the field

- Retaining the copyright to your article

Submit your next manuscript at $\gg$ springeropen.com 